\title{
ECONOMIC ORTHOGONALLY WELDED STIFFENING OF A UNIAXIALLY COMPRESSED STEEL PLATE
}

\author{
J. Farkas ${ }^{1}$ K. Jármai \\ University of Miskolc (Hungary) \\ E-mail: 19altfar@uni-miskolc.hu
}

\begin{abstract}
Stiffened plates and shells are the most characteristic structural types for optimization, since the number of stiffeners influences the cost significantly. A previous study has shown that a plate stiffened on one side with open section longitudinal ribs subject to uniaxial compression is not so economic than a cellular one. In the present article a plate orthogonally stiffened on one side is optimized. The orthogonal grid of ribs is more economic, since the transverse stiffeners increase significantly the overall buckling strength of the plate. Constraints on overall buckling and on stiffener induced failure are considered. The cost function includes material, welding and painting costs.
\end{abstract}

Keywords: plate buckling, stiffened plates, welded structures, structural optimization, fabrication cost, minimum cost design

\section{INTRODUCTION}

The main requirements of a modern engineering structure are the safety, fitness for production and economy. In the optimum design process the safety and producibility are fulfilled by design and fabrication constraints as well as the economy is achieved by the minimization of a cost function.

We have developed a cost calculation method mainly for welded structures, thus, we are able to determine the economy of a structural version and to compare the costs of these versions to each other [1]. Welded stiffened plates are applied in many steel structures. Our aim is to determine the most economic stiffening of a uniaxially compressed plate. Our structural model is a rectangular steel plate with simply supported edges, stiffened orthogonally by halved rolled I-section stiffeners welded to the base plate by double fillet welds. 
In our other study we have compared the costs of a plate stiffened on one side and a cellular plate both stiffened longitudinally and loaded by uniaxial compression [2]. Economic stiffening has been determined for an orthogonally stiffened plate loaded by bending [3].

In the optimization process the base plate thickness, as well as the number and height of stiffeners in both directions are sought, which fulfil the buckling constraints and minimize the cost function.

The applied mathematical method is the particle swarm algorithm.

The classic buckling stress is derived from the Huber's differential equation [4]. This stress is modified taking into account the effect of residual welding stresses and initial imperfections.

The cost function includes the material and fabrication (welding and painting) costs and is formulated according to the fabrication sequence. A series of rolled I-section stiffeners is selected according to the ARCELOR catalogue [5]. The flange width and thickness, as well as the web thickness are expressed by the section height using approximate formulae (see Appendix), thus, in the optimization only five unknowns should be determined.

\section{PROBLEM FORMULATION}

Determine the economic orthogonal stiffening of a rectangular plate with given main dimensions $a_{0}$ and $b_{0}$, subject to a uniformly distributed uniaxial compression of intensity $N_{x}$ (Figure 1), which fulfils the design and fabrication constraints and minimizes the cost function. Halved rolled I-section stiffeners are welded to the base plate by double fillet welds.

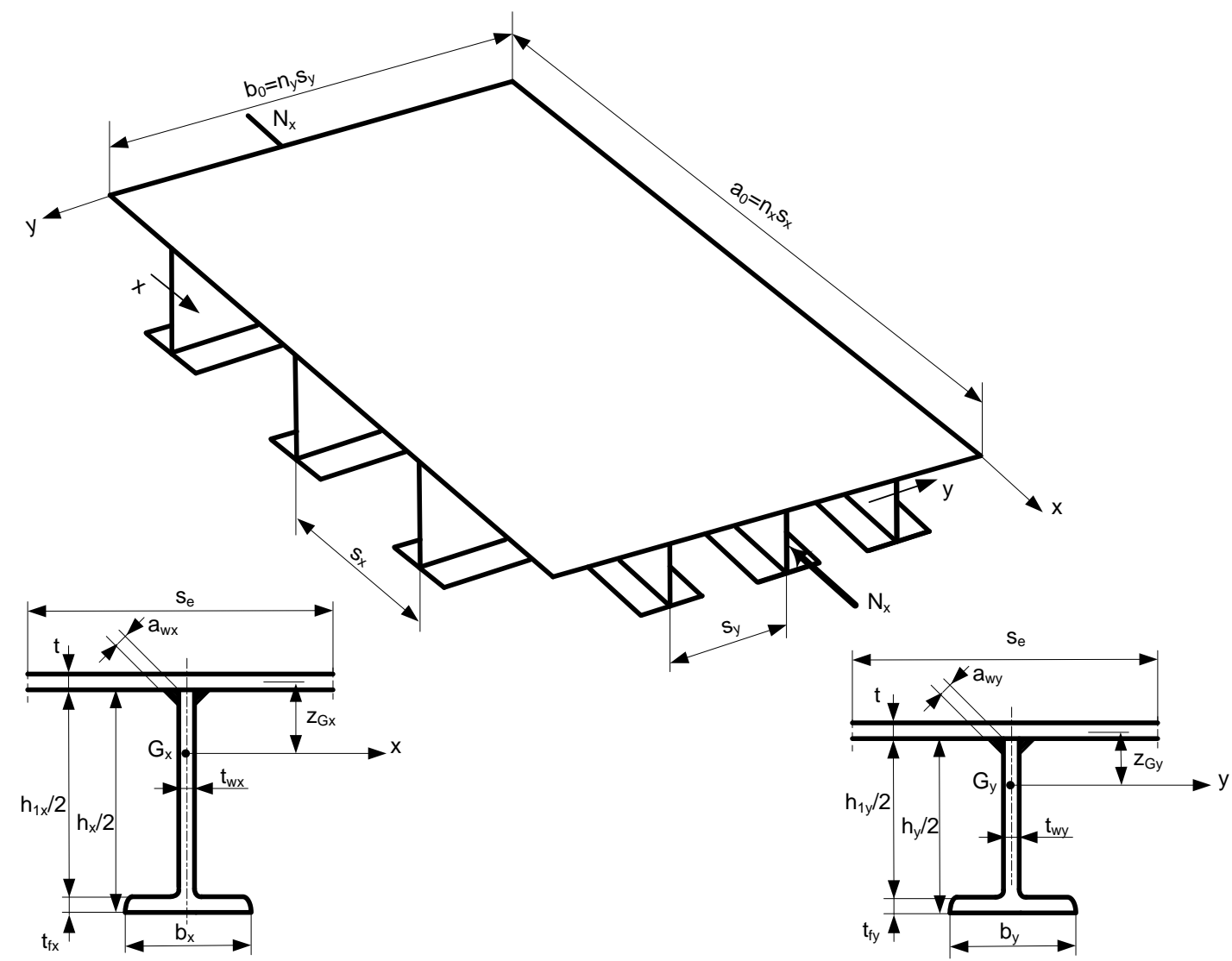

Figure 1 - Orthogonally stiffened plate loaded by uniaxial compression 
Numerical data (Figure 1): $a_{0}=24000, b_{0}=8000 \mathrm{~mm}, N_{x}=3 \times 10^{7}[\mathrm{~N}]$, steel yield stress $f_{y}=$ $355 \mathrm{MPa}$, elastic modulus $E=2.1 \times 10^{5} \mathrm{MPa}$, shear modulus $G=0.8 \times 10^{5}$, density $\rho=$ $7.85 \times 10^{-6} \mathrm{~kg} / \mathrm{mm}^{3}$, selected rolled I-sections UB profiles.

Unknowns to be optimized: base plate thickness $t$, sizes and number of stiffeners in both directions: $h_{y}, h_{x}, n_{y}, n_{x}$. Ranges of unknowns: $4<t<20 \mathrm{~mm}, 152<h<1016 \mathrm{~mm}, 4<n<n_{\max }$, $n_{\max }$ are determined by the following fabrication constraints:

$$
\frac{b_{0}}{n_{y}}-b_{y} \geq 300 \mathrm{~mm}, \quad \frac{a_{0}}{n_{x}}-b_{x} \geq 300 \mathrm{~mm} .
$$

The other dimensions of a halved rolled I-section are given by approximate functions of $h$ in Appendix.

$$
h_{1}=h-2 t_{f} .
$$

The discrete values of $h$ are as follows: 152.4, 177.8, 203.2, 257.2, 308.7, 353.4, 403.2, 454.6, 533.1, 607.6, 683.5, 762.2, 840.7, 910.4, $1016 \mathrm{~mm}$.

The maximum values of $n_{i}$ is given by the fabrication constraints Eq. (1).

The $n_{\max }$ values are given in the Table 1 .

Table 1. $n_{\max }$ - values for rolled I-sections, dimensions in $\mathrm{mm}$

\begin{tabular}{|l|l|l|l|l|l|l|l|l|l|l|l|l|l|l|}
\hline$h$ & 152.4 & 177.8 & 203.2 & 257.2 & 308.7 & 353.4 & 403.2 & 454.6 & 533.1 & 607.6 & 683.5 & 762.2 & 840.7 & 910.4 \\
\hline$b$ & 88.7 & 101.2 & 133.2 & 101.9 & 101.8 & 126.0 & 142.2 & 152.9 & 209.3 & 228.2 & 253.7 & 266.7 & 292.4 & 304.1 \\
\hline$n$ & 20 & 19 & 18 & 19 & 19 & 18 & 18 & 17 & 15 & 15 & 14 & 14 & 13 & 13 \\
\hline
\end{tabular}

\section{GEOMETRIC CHARACTERISTICS OF STIFFENERS}

Effective cross-sectional areas $(i=x, y)$

$$
A_{e i}=\frac{h_{1 i} t_{w i}}{2}+b_{i} t_{f i}+s_{e i} t, s_{y}=\frac{b_{0}}{n_{y}}, s_{x}=\frac{a_{0}}{n_{x}} .
$$

Effective plate widths in two directions for global plate buckling according to DNV [6]

$$
\begin{aligned}
& s_{e y}=\left(\frac{1.8}{\beta_{y}}-\frac{0.8}{\beta_{y}^{2}}\right) s_{y}, \quad s_{e x}=\left(\frac{1.8}{\beta_{x}}-\frac{0.8}{\beta_{x}^{2}}\right) s_{x} \\
& \beta_{y}=\frac{s_{y}}{t} \sqrt{\frac{f_{y}}{E}} \text { if } \quad \beta_{y} \geq 1 \\
& \beta_{y}=1 \quad \text { if } \quad \beta_{y}<1 \\
& \beta_{x}=\frac{s_{x}}{t} \sqrt{\frac{f_{y}}{E}} \text { if } \quad \beta_{x} \geq 1
\end{aligned}
$$


$\beta_{x}=1 \quad$ if $\quad \beta_{x}<1$

The distances of the gravity centres $\mathrm{G}_{\mathrm{i}}$

$$
z_{G i}=\frac{1}{A_{e i}}\left[\frac{h_{1 i} t_{w i}}{2}\left(\frac{h_{1 i}}{4}+\frac{t}{2}\right)+b_{i} t_{f i}\left(\frac{h_{i}+t-t_{f i}}{2}\right)\right],
$$

The moments of inertia

$$
I_{i}=s_{e i} z_{G i}^{2}+\frac{h_{1 i}^{3} t_{w i}}{96}+\frac{h_{1 i} t_{w i}}{2}\left(\frac{h_{1 i}}{4}+\frac{t}{2}-z_{G i}\right)^{2}+b_{i} t_{f i}\left(\frac{h_{i}+t-t_{f i}}{2}-z_{G i}\right)^{2}
$$

The bending stiffnesses

$$
B_{x}=\frac{E I_{y}}{s_{y}} ; B_{y}=\frac{E I_{x}}{s_{x}} .
$$

\section{DESIGN CONSTRAINTS}

Overall buckling constraint according to DNV [6]

$\sigma=\frac{N_{x}}{n_{y} A_{e y}} \leq \sigma_{c r}=\frac{f_{y 1}}{\sqrt{1+\lambda^{4}}}, f_{y 1}=\frac{f_{y}}{1.1}$

$\lambda=\sqrt{\frac{f_{y 1}}{\sigma_{E}}}, \sigma_{E}=\frac{N_{E} s_{y}}{A_{e y}}, N_{E}=\frac{\pi^{2}}{b_{0}^{2}}\left(B_{x} \frac{b_{0}^{2}}{a_{0}^{2}}+B_{y} \frac{a_{0}^{2}}{b_{0}^{2}}\right)$

It can be seen from the load-carrying capacity formula $N_{E}$ that, when $a_{0}>b_{0}$, to have a larger $N_{E}, B_{x}\left(h_{x}\right)$ should be larger than $B_{y}\left(h_{y}\right)$.

Constraint on stiffener induced failure according to DNV [6]

$$
s_{e y 1}=\left(1.1-0.1 \beta_{y}\right) s_{y}
$$

but $s_{\text {ey1.max }}=1$

$$
\begin{aligned}
& A_{e y 1}=\frac{h_{1} t_{w}}{2}+b t_{f}+s_{e y 1} t \\
& z_{G y 1}=\frac{h_{1} t_{w}}{2 A_{e y 1}}\left(\frac{h_{1}}{4}+\frac{t}{2}\right)+\frac{b t_{f}}{2 A_{e y 1}}\left(h_{1}+t-t_{f}\right)
\end{aligned}
$$




$$
\begin{aligned}
& I_{y 1}=s_{e y 1} t z_{G y 1}^{2}+\frac{h_{1}^{3} t_{w}}{96}+\frac{h_{1} t_{w}}{2}\left(\frac{h_{1}}{4}+\frac{t}{2}-z_{G y 1}\right)^{2}+I_{y 11} \\
& I_{y 11}=b t_{f}\left(\frac{h+t-t_{f}}{2}-z_{G y 1}\right)^{2} \\
& \sigma_{E x}=\frac{\pi^{2} E I_{y 1}}{A_{e y 1} s_{x}^{2}} \\
& \sigma_{E T}=\frac{A_{w}+A_{f}\left(\frac{t_{f}}{t_{w}}\right)^{2}}{A_{w f}} G\left(\frac{2 t_{w}}{h_{1}}\right)^{2}+\frac{3 x 2.6 \pi^{2} E I_{z}}{A_{w f} s_{x}^{2}}
\end{aligned}
$$

where

$$
\begin{aligned}
& A_{w}=\frac{h_{1} t_{w}}{2}, A_{f}=b t_{f}, A_{w f}=A_{w}+3 A_{f}, I_{z}=\frac{b^{3} t_{f}}{12} \\
& \lambda_{T}=\sqrt{\frac{f_{y}}{\sigma_{E T}}} \\
& \sigma_{T}=\frac{f_{y 1}}{\phi_{T}+\sqrt{\phi_{T}^{2}-\lambda_{T}^{2}}}, \phi_{T}=0.5\left(1+\mu_{T}+\lambda_{T}^{2}\right) \\
& \mu_{T}=0.007\left(\lambda_{T}-0.6\right) \\
& \lambda_{S}=\sqrt{\frac{\sigma_{k}}{\sigma_{E x}}}
\end{aligned}
$$

where

$$
\begin{array}{ll}
\sigma_{k}=f_{y} \quad \text { if } \quad \lambda_{T}<0.6 \\
\sigma_{k}=\sigma_{T} \text { if } \quad \lambda_{T} \geq 0.6
\end{array}
$$

The constraint is formulated as

$$
\sigma_{1}=\frac{N_{x}}{n_{y} A_{e y 1}} \leq \sigma_{a c r}=\frac{\sigma_{k}}{\phi+\sqrt{\phi^{2}-\lambda_{S}^{2}}}
$$

where 
$\phi=0.5\left(1+\mu+\lambda_{S}^{2}\right)$

$\mu=\frac{\delta z_{t} A_{e y 1}}{I_{y 1}} \quad$ if $\quad \lambda_{T}<0.6$

$\mu=\frac{2.3 \delta z_{t} A_{e y 1}}{I_{y 1}}$ if $\quad \lambda_{T} \geq 0.6$

$\delta=0.0015 s_{x}$

$z_{t}=z_{G y 1}+\frac{t_{f}}{2}$

\section{COST FUNCTION}

The cost function includes the cost of material, assembly, welding as well as painting and is formulated according to the fabrication sequence.

The cost of material

$$
K_{M}=k_{M} \rho V_{2} ; k_{M}=1.0 \$ / \mathrm{kg} \text {. }
$$

Welding of the base plate from butt welds ( 3 in direction of $a_{0}$ and 3 in direction of $b_{0}$ ) (SAW - submerged arc welding) [1]:

The fabrication cost factor is taken as $k_{F}=1.0 \$ / \mathrm{min}$, the factor of complexity of the assembly $\Theta_{W}=2$ :

$$
\begin{aligned}
& K_{0}=k_{F}\left[\Theta_{W} \sqrt{16 \rho V_{0}}+1.3 C_{W} t^{n}\left(3 a_{0}+3 b_{0}\right)\right], \\
& \text { for } t<11 \quad V_{0}=a_{0} b_{0} t, \\
& \text { for } t \geq 11 \quad C_{W}=0.1346 \times 10^{-3} ; n=2, \\
& C_{W}=0.1033 \times 10^{-3} ; n=1.904 .
\end{aligned}
$$

Welding $\left(n_{x}-1\right)$ stiffeners to the base plate in $y$ direction with double fillet welds (GMAW-C gas metal arc welding with $\mathrm{CO}_{2}$ ):

$$
K_{W 1}=k_{F}\left\lfloor\Theta_{W} \sqrt{n_{x} \rho V_{1}}+1.3 \times 0.3394 \times 10^{-3} a_{w x}^{2} 2 b_{0}\left(n_{x}-1\right)\right\rfloor,
$$

$a_{W x}=0.4 t_{w x}$ but $a_{w x \cdot \min }=3 \mathrm{~mm}$,

$$
V_{1}=a_{0} b_{0} t+\left(\frac{h_{1 x} t_{w x}}{2}+b_{x} t_{f x}\right) b_{0}\left(n_{x}-1\right)
$$


Welding of $\left(n_{y}-1\right)$ stiffeners to the base plate in $x$ direction with double fillet welds. These stiffeners should be interrupted and welded with fillet welds to the stiffeners in the $y$ direction.

$$
\begin{gathered}
\left.K_{W 2}=k_{F} \mid \Theta_{W} \sqrt{\left(n_{y} n_{x}-n_{x}+1\right) \rho V_{2}}+1.3 \times 0.3394 \times 10^{-3} a_{w y}^{2} 2 a_{0}\left(n_{y}-1\right)+T_{1}\right\rfloor, \\
T_{1}=1.3 \times 0.3394 \times 10^{-3} a_{w y}^{2} 4\left(n_{y}-1\right)\left(n_{x}-1\right)\left(\frac{h_{1 y}}{2}+b_{y}\right),
\end{gathered}
$$

$a_{W y}=0.4 t_{w y}$ but $a_{W y \cdot \min }=3 \mathrm{~mm}$,

$V_{2}=V_{1}+\left(\frac{h_{1 y} t_{w y}}{2}+b_{y} t_{f y}\right) a_{0}\left(n_{y}-1\right)$

Painting

$$
K_{P}=k_{P} \Theta_{P} S_{P}
$$

$k_{P}=14.4 \times 10^{-6} \$ / \mathrm{mm}^{2}, \Theta_{\mathrm{P}}=2$,

Surface to be painted

$$
S_{P}=2 a_{0} b_{0}+a_{0}\left(n_{y}-1\right)\left(h_{1 y}+2 b_{y}\right)+b_{0}\left(n_{x}-1\right)\left(h_{1 x}+2 b_{x}\right)
$$

The total cost

$$
K=K_{M}+K_{0}+K_{W 1}+K_{W 2}+K_{P}
$$

\section{THE PARTICLE SWARM OPTIMIZATION (PSO) ALGORITHM}

The general optimization problem to be considered here is therefore:

$$
\underset{\text { w.r.t. } \boldsymbol{x}}{\operatorname{minimize}} f(\boldsymbol{x}), \boldsymbol{x}=\left[x_{1}, x_{2}, \mathrm{~K} x_{n}\right]^{T} \in R^{n},
$$

subject to the inequality and equality constraints:

and side constraints:

$$
\begin{array}{ll}
g_{j}(\boldsymbol{x}) \leq 0, & j=1,2, \mathrm{~K}, m \\
h_{j}(\boldsymbol{x})=0, & j=1,2, \mathrm{~K}, r
\end{array}
$$

$$
x_{i}^{l} \leq x_{i} \leq x_{i}^{u}, \quad i=1,2, \ldots, n,
$$

where $f(\boldsymbol{x}), g_{j}(\boldsymbol{x})$ and $h_{j}(\boldsymbol{x})$ are scalar functions of the real column vector $\boldsymbol{x}$. For generality equality constraints, $h_{j}(\boldsymbol{x})=0, j=1,2, \ldots, r$ are also specified, although they are not explicitly imposed in this study. The optimum solution is denoted by $x^{*}$ with associate optimum function value $f\left(x^{*}\right)$. 
Particle Swarm Optimization (PSO) techniques belong to a relatively new class of evolutionary based search procedures that may be used to find the optimum solution $x^{*}$ of the general optimization problem. The original PSO algorithm, proposed by Kennedy and Eberhardt [7], was inspired by the modelling of the social behaviour patterns of organisms that live and interact within large groups. In particular, PSO incorporates swarming behaviours observed in flocks of birds, schools of fish, or swarms of bees.

A PSO algorithm is easy to implement in most programming languages, since the core of the program can be written in a few lines of code. It has been proven to be both fast and effective, when applied to a diverse set of optimization problems. PSO algorithms are especially useful for parameter optimization in continuous, multi-dimensional search spaces [8].

In performing a search in the multi-dimensional space associated with the optimization problem of the form $(39,40)$, the PSO technique assigns direction vectors and velocities to each member (particle) of the swarm at their current positions. Each particle then "moves" or "flies" through the search space according to the particle's assigned velocity vector, which may be influenced by the directions and velocities of other particles in its neighbourhood. These localized interactions with neighbouring particles, propagate through the entire "swarm" of particles and results in the swarm as a whole moving to regions of the space closer to the solution of problem $(39,40)$.

The extent to which a particular particle influences other particles is determined by its socalled "fitness" along its trajectory of candidate solution points. The "fitness" is a measure assigned to each potential solution, and it indicates how good a particular candidate solution is relative to all other solution points. Hence, an evolutionary idea of "survival of the fittest" (in the sense of Darwinian evolution) comes into play, as well as a social behaviour component through a "follow the local leader" effect and emergent pattern formation [9].

\section{OPTIMIZATION AND RESULTS}

The optima of unknowns are as follows.

$h_{y}=353.4, h_{x}=533.1, t=12 \mathrm{~mm}, n_{y}=14, n_{x}=5$. The constraints are fulfilled, since $\sigma=292$ $<\sigma_{\mathrm{cr}}=299 \mathrm{MPa}$ and $\sigma_{\mathrm{S}}=230<\sigma_{\mathrm{acr}}=243 \mathrm{MPa}$. The minimum cost is $K=51087 \$$.

It should be mentioned that the calculation of the critical buckling stress (Eqs 8, 9) according to DNV [6] takes into account the effect of residual welding stresses and distortions. The considered measure of welding distortion is about $L / 1000$ where $L$ is the span length. This distortion can be approximately calculated using our formulae published earlier [10].

For the optimum solution with fillet weld size $a_{w}=4 \mathrm{~mm}$, for double fillet welds taking a factor of 1.5: $Q_{T}=1.5 \times 59.5 a_{w}^{2}=1428 \mathrm{~J} / \mathrm{mm}, z_{G x}^{\prime}=100.36-6=94.36 \mathrm{~mm}$,

$I_{x}=1.658 \times 10^{8} \mathrm{~mm}^{4}, b_{0}=8000 \mathrm{~mm}$,

$C=\frac{0.844 \times 10^{-3} Q_{T} z_{G}^{\prime}}{I_{x}}=0.6859 \times 10^{-6} 1 / \mathrm{mm}, f=\frac{C b_{0}^{2}}{8}=5.5<8=\frac{b_{0}}{1000}$. Thus, the calculation of the critical buckling stress gives safe values. 


\section{CONCLUSIONS}

Orthogonally stiffened plates are important elements of welded structures, thus their minimum cost design influences the economy of these structures significantly. The basic formula for overall buckling strength shows that the transverse stiffening increases the plate strength in a great measure.

In the optimization process the height and number of halved rolled I-section stiffeners as well as the base plate thickness is sought, which fulfil the design constraints and minimize the cost function. Both the global buckling and the stiffener induced failure constraints are active.

The particle swarm algorithm has been proved to be efficient in finding the optima.

An approximate calculation shows that the deflections caused by the shrinkage of longitudinal welds are smaller than the deflection taking into account as initial imperfections in the buckling strength formulae.

\section{ACKNOWLEDGEMENTS}

The research work was supported by the Hungarian Scientific Research Foundation grants OTKA T38058, T37941 and the Öveges József scholarship.

\section{REFERENCES}

[1] Farkas J., Jármai K.: Economic design of metal structures, Rotterdam, Millpress, 2003.

[2] Farkas J., Jármai K.: Optimum design and cost comparison of a welded plate stiffened on one side and a cellular plate both loaded by uniaxial compression, Welding in the World, 2006, Vol.50, No.3-4. pp. 45-51.

[3] Jármai K., Farkas J., Groenwold A.: Economic welded stiffening of a steel plate loaded by bending. IIW Regional Welding Congress, Stellenbosch, South Africa, 2006. CD-Rom.

[4] Timoshenko S.P., Gere J.M.: Theory of elastic stability, $2^{\text {nd }}$ ed. New York-TorontoLondon, McGraw Hill, 1961.

[5]. Profil ARBED Sales Program. Structural Shapes. ARCELOR Long Commercial, 2001.

[6] Det Norske Veritas (DNV): Buckling strength analysis. Classification Notes No.30.1. Høvik, Norway, 1995.

[7] Kennedy J., Eberhardt R.: Particle swarm optimization, Proc. Int. Conf. on Neural Networks, Piscataway, NJ, USA, 1995, pp. 1942-1948.

[8] Jármai K.: Particle swarm method as a new tool for structural optimization, Journal of Computational and Applied Mechanics, 2005, Vol. 6, No. 2. pp. 207-226, Miskolc University Press. 
[9] Jármai K., Snyman J.A., Farkas J.: Minimum cost design of a welded orthogonally stiffened cylindrical shell, Journal of Computers and Structures, 2006, Vol. 84, pp. 787797.

[10] Farkas J., Jármai K.: Analysis of some methods for reducing residual beam curvatures due to weld shrinkage, Welding in the World, 1998, Vol. 41, No.4, pp.385-398.

\section{APPENDIX}

Approximate formulae for UB profile dimensions

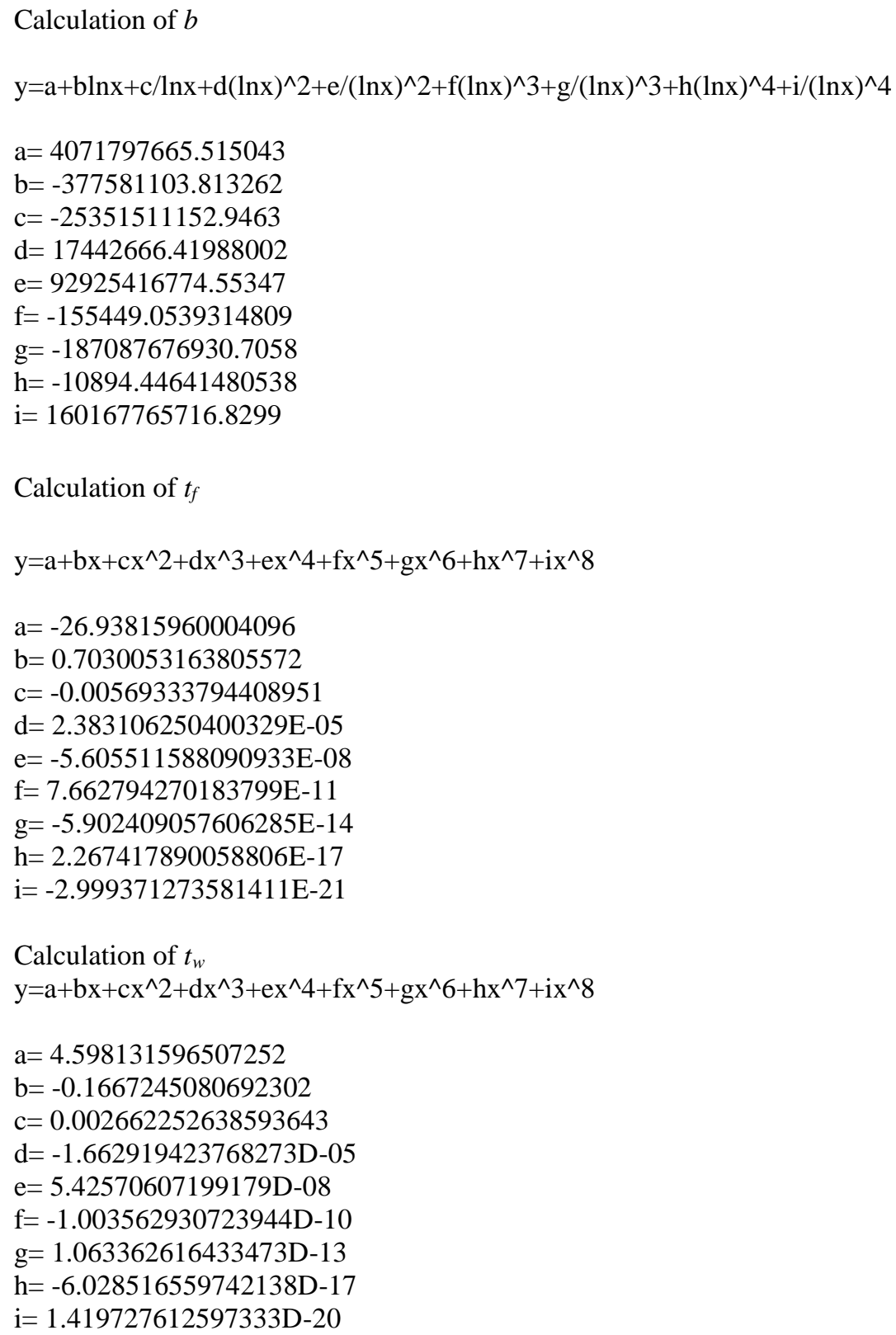

